\title{
Phase Equilibria of Quasi-Ternary Systems Consisting of Multicomponent Polymers in a Binary Solvent Mixture I. Theoretical Basis and Suitable Choice of Solvent 1 and Solvent 2
}

\author{
Kenji KAMIDE, Shigenobu MATSUdA, and Yukio MiYAZAKI \\ Textile Research Laboratory, Asahi Chemical Industry Company, Ltd., \\ Takatsuki, Osaka 569, Japan
}

(Received January 26, 1984)

\begin{abstract}
A theory of the phase equilibrium of a quasi ternary system consisting multicomponent polymers dissolved in binary solvent mixture was proposed as an extension of that for the quasi-binary system established so far by Kamide et al. In this study, the concentration- and molecular weight-dependences of three $\chi$ parameters, $\chi_{12}, \chi_{13}$, and $\chi_{23}$ are ignored, where the suffixes 1,2 , and 3 denote solvent 1 , a good solvent, solvent 2 , a poor solvent, and a polymer, respectively. A computer simulation technique was established on the basis of the theory. From computer experiments on solutions of the polymer with the Schulz-Zimm type molecular weight distribution (the ratio of weight- to number-average molar volume ratio of the polymer to the solvents, $X_{w} / X_{n}=2.0$ and $X_{w}=300$ ), a suitable choice of solvent 1 and solvent 2 , yielding the phase separation under given conditions, is discussed. It was found that the polymer volume fraction in a polymer-rich phase $v_{p(2)}$, the partition coefficient $\sigma$, the phase volume ratio $R$ and the molecular weight distribution of the polymers in both phases are predominantly controlled by $\chi_{23}$, if $\chi_{23}<1.0$ and are influenced by $\chi_{12}$ and $\chi_{23}$, rather than $\chi_{13}$, if $\chi_{23}>1.2$.
\end{abstract}

KEY WORDS Quasi-Ternary Polymer Solution / Multicomponent Polymers-Solvent-Nonsolvent System / Phase Equilibrium / Computer Simulation / Polymer-Solvent Interaction Parameter / Partition Coefficient / Phase Volume Ratio /

Since 1968, Kamide and his coworkers ${ }^{1}$ and Koningsveld et $a .^{2}$ have independently and rigorously studied the two phase equilibrium, at constant temperature and pressure, of multicomponent polymers, all belonging to chemical homologues, dissolved in a single solvent. These are quasi two component systems (i.e., quasi-binary systems) and the authors succeeded in clarifying their fundamental features.

The two phase equilibrium phenomena of a quasi-ternary system consisting of multicomponent polymers, solvent, and nonsolvent are used not only for molecular weight fractionation by solubility difference, but also extensively for many industrial processes, such as the wet-spinning of regenerated and synthetic fibers, solvent-casting of micro-porous membranes and polymer coating. The quasi-ternary system thus warrants study so as to establish some scientific basis for it.

The phase separation phenomena of a single component polymer/solvent/nonsolvent system (i.e., rigorous ternary system) have been energetically studied from as early as the late 1940 s' to 1950 s'. Even for this relatively simple system, all two phase equilibrium calculations at constant temperature and pressure carried out in the literature are far from rigorousness and based on the following crude assumptions: (a) a solvent mixture can be approximated as a "single solvent" (Flory, ${ }^{3}$ Scott $^{4,5}$ ), (b) polymer molecular weight is infinite (Scott, ${ }^{4,5}$ Nakagaki-Sunada ${ }^{6}$ ), (c) among three thermo- 
dynamic interaction parameters between solvent (1), nonsolvent (2), and polymer (3), $\chi_{12}$, $\chi_{23}, \chi_{13}, \chi_{12}=\chi_{13}$, and $\chi_{23}=0$ hold (Tompa, ${ }^{7}$ Nakagaki and Sunada ${ }^{6}$ ), and (d) polymer does not exist in polymer-lean phase (KrigbaumCarpenter, ${ }^{8}$ Suh-Liou ${ }^{9}$ ).

Assumptions (a) - (d) are not generally acceptable and hence the conclusions obtained by them cannot be regarded as having general applicability. The most reliable calculation on the plait points of a ternary system were made by Nakagaki and Sunada, ${ }^{6}$ who numerically calculated the relationship between the interaction parameters between solvent-polymer $\left(\chi_{13}\right)$, solvent-nonsolvent $\left(\chi_{12}\right)$, and nonsolvent-polymer $\left(\chi_{23}\right)$ pairs and the plait points for precipitation on the basis of Scott's critical point equation.

Theoretical study on the phase equilibrium of multicomponent polymers/solvent/nonsolvent system has been carried out only on a small scale since Münster's first study on a quasi-ternary system. Okamoto ${ }^{11}$ calculated the threshold point of the same system and Koningsveld ${ }^{12}$ demonstrated, on the basis of Flory-Huggins high polymer solution theory, some coexistence curves, using rigorous calculation techniques, but he showed none of the details for this or phase characteristics.

In spite of its scientific and industrial importance, the study of the phase equilibrium of the quasi-ternary system, is still at very primitive stage. This prompted to carry out a study, as an extention of the phase equilibrium theory, apply the simulation techniques of the quasibinary systems to the quasi-ternary system, and show various calculation results obtained by this theory and simulation techniques.

\section{THEORETICAL BACKGROUND}

We started from the Flory-Huggins theory of a polymer solution, assuming that (a) thermodynamic interaction parameters $\chi_{i j}(i \neq j$ and $i, j=1,2,3)$ are independent of polymer molecular weight and concentration, (b) the molar volume of solvent 1 (usually good solvent) is the same as that of solvent 2 (usually nonsolvent), (c) polymer, solvents 1 and 2 are volumetrically additive, and (d) the densities of polymer and solvents 1 and 2 are the same (=unity). Note that these assumptions are not absolutely necessary and consequently do not limit the theory. In this respect, our theory seems better than those proposed so far.

Consider a quasi-ternary system consisting of solvents 1 and 2 and multicomponent nonelectrolyte polymers at constant temperature and pressure. The partial molar free energy of these solvents and the $X$-mer, $\Delta \mu_{1}, \Delta \mu_{2}$, and $\Delta \mu_{x}$, constituting the systems are given according to the Flory-Huggins theory by the following relations respectively:

$$
\begin{aligned}
& \Delta \mu_{1}=\tilde{R} T\left\{\ln v_{1}+\left(1-\frac{1}{X_{n}}\right) v_{p}+\chi_{12} v_{2}^{2}+\chi_{13} v_{p}^{2}+\left(\chi_{12}+\chi_{13}-\chi_{23}\right) v_{2} v_{p}\right\} \\
& \Delta \mu_{2}=\tilde{R} T\left\{\ln v_{2}+\left(1-\frac{1}{X_{n}}\right) v_{p}+\chi_{12}\left(1-v_{2}\right)^{2}+\chi_{13} v_{p}^{2}-\left(\chi_{12}+\chi_{13}-\chi_{23}\right)\left(v_{p}-v_{2} v_{p}\right)\right\} \\
& \Delta \mu_{x}=\tilde{R} T\left\{\ln v_{x}-(X-1)+X\left(1-\frac{1}{X_{n}}\right) v_{p}\right. \\
& \left.\quad+X \chi_{12} v_{2}^{2}+X \chi_{13}\left(1-v_{p}\right)^{2}-X\left(\chi_{12}+\chi_{13}-\chi_{23}\right)\left(v_{2}-v_{2} v_{p}\right)\right\}
\end{aligned}
$$

where $X$ is the molar volume ratio of the polymer to solvent 1 or 2 and $X$ reduces to the degree of polymerization when the molar vol- ume of the repeating unit is the same as that of solvents. $\tilde{R}$ is the gas constant, $T$, the temperature, $v_{1}$ and $v_{2}$, the volume fractions of the 


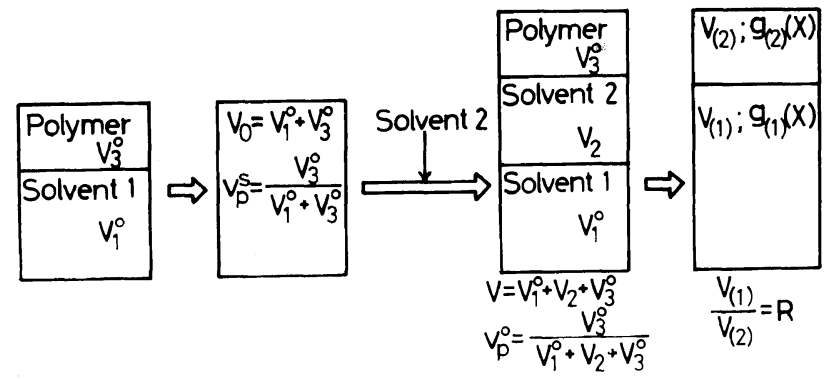

Figure 1. Phase equilibrium experiment of a quasi-ternary system; $V_{1}^{0}$, volume of solvent $1 ; V_{2}$, volume of solvent $2 ; V_{3}^{0}$, volume of polymer; $V_{0}=V_{1}^{0}+V_{3}^{0} ; v_{p}^{s}$, polymer volume fraction of the starting solution; $V$, total volume of the solution at phase equilibrium; $v_{p}^{0}$, polymer volume fraction of the solution at phase equilibrium; $R$, phase volume ratio; $V_{(1)}$, volume of polymer-lean phase; $V_{(2)}$, volume of polymer-rich phase; $g_{(1)}(X)$, MWD of the polymer in the polymer-lean phase; $g_{(2)}(X)$, MWD of the polymer in the polymer-rich phase.

solvent 1 and solvent 2 , respeceively, $v_{p}$, the total polymer volume fraction $\left(\equiv \sum v_{x}, v_{x}\right.$ is volume fraction of the $X$-mer), and $X_{n}$ is the number-average $X$. Here, $\Delta \mu_{x}$ at $X=1$ and at $X=2$ is thus represented so as to distinguish it from $\Delta \mu_{1}$ and $\Delta \mu_{2}$, by $\Delta \mu_{x=1}$ and $\Delta \mu_{x=2}$, respectively. The details for the derivation of eq $1-3$ are well-known and need not be given here.

Under the two-phase equilibrium of the quasi-ternary system at constant temperature and pressure, for following condition should be satisfied.

$$
\begin{aligned}
& \Delta \mu_{1(1)}=\Delta \mu_{1(2)} \\
& \Delta \mu_{2(1)}=\Delta \mu_{2(2)} \\
& \Delta \mu_{x(1)}=\Delta \mu_{x(2)}
\end{aligned}
$$

The suffixes (1) and (2) in eq 4 denote the polymer-lean and -rich phases, respectively. Note that eq $4 \mathrm{c}$ should hold for all polymer components, differing $X$.

The partition coefficient $\sigma$ is defined by eq 5

$$
\sigma=\frac{1}{X} \ln \frac{v_{x(2)}}{v_{x(1)}}
$$

The ratio $v_{x(2)} / v_{x(1)}$ can be calculated by eq 3 and $4 \mathrm{c}$ and eq 5 may thus be rearranged as,

$$
\begin{aligned}
\sigma= & \left(1-\frac{1}{X_{n(1)}}\right) v_{p(1)}-\left(1-\frac{1}{X_{n(2)}}\right) v_{p(2)} \\
& +\chi_{12}\left(v_{2(1)}^{2}-v_{2(2)}^{2}\right)+\chi_{13}\left(1-v_{p(1)}\right)^{2} \\
& -\left(1-v_{p(2)}\right)^{2}-\left(\chi_{12}+\chi_{13}-\chi_{23}\right)\left\{\left(v_{2(1)}-v_{2(2)}\right)\right. \\
& \left.-\left(v_{2(1)} v_{p(1)}-v_{2(2)} v_{p(2)}\right)\right\}
\end{aligned}
$$

Substitution of eq 1 into eq 4 a gives

$$
\begin{aligned}
& \ln \frac{v_{1(2)}}{v_{1(1)}}-\left(v_{p(1)}-v_{p(2)}\right)+\left(\frac{v_{p(1)}}{X_{n(1)}}-\frac{v_{p(2)}}{X_{n(2)}}\right) \\
& -\chi_{12}\left(v_{2(1)}^{2}-v_{2(2)}^{2}\right)-\chi_{13}\left(v_{p(1)}^{2}-v_{p(2)}^{2}\right) \\
& -\left(\chi_{12}+\chi_{13}-\chi_{23}\right)\left(v_{2(1)} v_{p(1)}-v_{2(2)} v_{p(2)}\right)=0
\end{aligned}
$$

Combining eq 2 and $4 \mathrm{~b}$ yields

$$
\begin{aligned}
& \ln \frac{v_{2(2)}}{v_{2(1)}}-\left(v_{p(1)}-v_{p(2)}\right)+\left(\frac{v_{p(1)}}{X_{n(1)}}-\frac{v_{p(2)}}{X_{n(2)}}\right) \\
& -\chi_{12}\left\{\left(1-v_{2(1)}\right)^{2}-\left(1-v_{2(2)}\right)^{2}\right\} \\
& -\chi_{13}\left(v_{p(1)}^{2}-v_{p(2)}\right)+\left(\chi_{12}^{2}+\chi_{13}-\chi_{23}\right) \\
& \quad \times\left\{\left(v_{p(1)}-v_{p(2)}\right)-\left(v_{2(1)} v_{p(1)}-v_{2(2)} v_{p(2)}\right)\right\}=0
\end{aligned}
$$

A schematic representation of the computer experiment on the phase equilibrium of a quasi-ternary solution is shown in Figure 1. The computer experiment was carried out in the following manner. Represent the param- 
eters $v_{p(1)}, v_{p(2)}, v_{1(1)}$, and $v_{1(2)}$ in eq $6-8$ in terms of $v_{2(1)}$ and $v_{2(2)}$. Solve eq 7 and 8, modified in this manner, under the given conditions of the phase separation to obtain $v_{2(1)}$ and $v_{2(2)}$. Put these values into modified eq 6 to calculate $\sigma$.

We express the normalized distribution function of $X$ for the original polymer (hereafter, we simply refer to it as the molecular weight distribution) as $g_{0}(X)$. Dissolve a unit weight of the polymer (whose volume is equal to the specific volume $V_{3}^{0}(\equiv 1)$ ) in a volume of the solvent $1, V_{1}^{0}$, to give a solution of the polymer volume fraction $v_{p}^{\mathrm{s}}\left(=V_{3}^{0} /\left(V_{1}^{0}+\right.\right.$ $\left.V_{3}^{0}\right)$ ). To this solution, a given volume of nonsolvent (solvent 2) $V_{2}$ is added to bring about the two phase separation. The polymer volume fraction, at which the two phase separation occurs, $v_{p}^{0}$, is given by

$$
\begin{aligned}
v_{p}^{0} & =V_{3}^{0} / V=V_{3}^{0} /\left(V_{1}^{0}+V_{2}+V_{3}^{0}\right) \\
& =1 /\left(V_{1}^{0}+V_{2}+1\right)
\end{aligned}
$$

Here, $V$ is the volume of the quasi-ternary solution. The volumes of the polymer-lean and polymer-rich phases, $V_{(1)}$ and $V_{(2)}$ are represented by eq $10 \mathrm{a}$ and $10 \mathrm{~b}$, respectively.

$$
\begin{aligned}
& V_{(1)}=R V /(R+1) \\
& V_{(2)}=V /(R+1)
\end{aligned}
$$

with

$$
R=V_{(1)} / V_{(2)}
$$

$R$ is the phase volume ratio. The weight fraction of the polymers partitioned into the polymer-lean and polymer-rich phases to the polymer dissolved in the initial solution are represented by $\rho_{s}\left(\equiv 1-\rho_{p}=\sum_{x} g_{0}(X)-\rho_{p}\right)$ and $\rho_{p}$, respectively. We denote the amount of $X$ mer remaining in the polymer-rich phase by $g_{(2)}(X)$ and that in the polymer-lean phase by $g_{(1)}(X)$ and eq 12 holds:

$$
\sum_{x} g_{0}(X)=\sum_{x} g_{(1)}(X)+\sum_{x} g_{(2)}(X)
$$

Here, $g_{(1)}(X)$ and $g_{(2)}(X)$ are unnormalized molecular weight distributions. The total polymer volume fraction in both phases, $v_{p(1)}$ and $v_{p(2)}$, can be represented by summing the volume fractions of the $X$-mer in the two phases, $v_{x(1)}$ and $v_{x(2)}$;

$$
\begin{aligned}
& v_{p(1)}=\sum_{x} v_{x(1)}=\sum_{x} g_{(1)}(X) / V_{(1)} \\
& v_{p(2)}=\sum_{x} v_{x(2)}=\sum_{x} g_{(2)}(X) / V_{(2)}
\end{aligned}
$$

From eq 5, 12, and 13 we obtain,

$$
\begin{aligned}
& g_{(1)}(X)=\frac{R}{R+\exp (\sigma X)} g_{0}(X) \\
& g_{(2)}(X)=\frac{\exp (\sigma X)}{R+\exp (\sigma X)} g_{0}(X)
\end{aligned}
$$

Combination of eq 10 and 14 yields

$$
\begin{aligned}
& v_{x(1)}(X)=\frac{R+1}{V(R+\exp (\sigma X))} g_{0}(X) \\
& v_{x(2)}(X)=\frac{(R+1) \exp (\sigma X)}{V(R+\exp (\sigma X))} g_{0}(X)
\end{aligned}
$$

We obtain the total volume $V$ of the two phases by adding the volumes of solvent $1, V_{1}^{0}$, solvent $2, V_{2}$, and the polymer, $V_{3}^{0}$ in the form:

$$
V=V_{0}^{0}+V_{2}+V_{3}^{0}=\frac{(R+1)\left(\sum_{x} g_{0}(X)+V_{1}^{0}\right)}{R\left(1-v_{2(1)}\right)+\left(1-v_{2(2)}\right)}
$$

Here, $V_{1}^{0}$ and $V_{3}^{0}$ are kept constant throughout the phase separation and the suffix 0 is attached to them. From eq 13, 14, and 15, we obtain

$$
\begin{aligned}
& v_{p(1)}=\left(\rho_{s} / R V_{0}\right)\left\{(R+1)-\left(R v_{2(1)}+v_{2(2)}\right)\right\} \\
& v_{p(2)}=\left(\rho_{p} / V_{0}\right)\left\{(R+1)-\left(R v_{2(1)}+v_{2(2)}\right)\right\}
\end{aligned}
$$

with

$$
V_{0}=V_{1}^{0}+V_{3}^{0}=V_{1}^{0}+\sum_{x} g_{0}(X)
$$

The volume fraction of solvent 1 in the two phases, $v_{1(1)}$ and $v_{1(2)}$ are defined by 


$$
\begin{aligned}
& v_{1(1)}=1-v_{2(1)}-v_{p(1)} \\
& v_{1(2)}=1-v_{2(2)}-v_{p(2)}
\end{aligned}
$$

Substitution of eq 17 into eq 19 gives,

$$
\begin{aligned}
v_{1(1)}=1 & -\left(\rho_{s} / R V_{0}\right)(R+1) \\
& -\left(1-\rho_{s} / V_{0}\right) v_{2(1)}+\left(\rho_{s} / R V_{0}\right) v_{2(2)} \\
v_{1(2)}=1 & -\left(\rho_{p} / V_{0}\right)(R+1) \\
+ & \left(\rho_{p} R / V_{0}\right) v_{2(1)}-\left(1-\rho_{p} / V_{0}\right) v_{2(2)}
\end{aligned}
$$

Then, $v_{1(1)}, v_{1(2)}, v_{p(1)}, v_{p(2)}$, and $X_{n(1)}$ in eq 6-8 can be expressed in terms of $V_{0}, \rho_{p}$ (or $\left.\rho_{s}\right), R$, $X_{n}^{0}, X_{n(2)}, v_{2(1)}$, and $v_{2(2)}$, by using eq $17-20$. The results are summarized as follows.

$$
\begin{aligned}
& \sigma=\left\{\frac{2 \chi_{13}-1}{V_{0}}\left(\rho_{p}-\frac{\rho_{s}}{R}\right)\right. \\
&+\frac{1}{R V_{0}}\left((R+1) \frac{\rho_{p}}{X_{n(2)}}-\frac{1}{X_{n}^{0}}\right) \\
&-\quad \frac{\chi_{13}}{V_{0}^{2}}\left(\rho_{p}^{2}-\frac{\rho_{s}^{2}}{R^{2}}\right) \\
& \quad \times\left\{(R+1)-\left(R v_{2(1)}+v_{2(2)}\right)\right\} \\
&\left.-\frac{\chi_{12}+\chi_{13}-\chi_{23}}{V_{0}}\left(\rho_{p} v_{2(2)}-\frac{\rho_{s}}{R} v_{2(1)}\right)\right\} \\
& \quad \times\left((R+1)-\left(R v_{2(1)}+v_{2(2)}\right)\right) \\
&+\left(\chi_{12}+\chi_{13}-\chi_{23}\right)\left(v_{2(2)}-v_{2(1)}\right) \\
&- \chi_{12}\left(v_{2(2)}^{2}-v_{2(1)}^{2}\right)
\end{aligned}
$$

$\ln \frac{V_{0}-\rho_{p}(R+1)+\rho_{p} R v_{2(1)}-\left(V_{0}-\rho_{p}\right) v_{2(2)}}{V_{0}-\rho_{s}\left(\frac{R+1}{R}\right)-\left(V_{0}-\rho_{s}\right) v_{2(1)}+\rho_{s} v_{2(2)} / R}$

$+\left\{\frac{1}{V_{0}}\left(\rho_{p}-\frac{\rho_{s}}{R}\right)-\frac{1}{R V_{0}}\left((R+1) \frac{\rho_{p}}{X_{n(2)}}-\frac{1}{X_{n}^{0}}\right)\right.$

$+\frac{\chi_{13}}{V_{0}^{2}}\left(\rho_{p}^{2}-\frac{\rho_{s}^{2}}{R^{2}}\right)\left((R+1)-\left(R v_{2(1)}+v_{2(2)}\right)\right)$

$\left.+\frac{\chi_{12}+\chi_{13}-\chi_{23}}{V_{0}}\left(\rho_{p} v_{2(2)}-\frac{\rho_{s}}{R} v_{2(1)}\right)\right\}$

$\times\left((R+1)-\left(R v_{2(1)}+v_{2(2)}\right)\right)$

$+\chi_{12}\left(v_{2(2)}^{2}-v_{2(1)}^{2}\right)=0$ and

$\ln \frac{V_{2(2)}}{v_{2(1)}}+\left\{\frac{1}{V_{0}}\left(\rho_{p}-\frac{\rho_{s}}{R}\right)\right.$

$+\frac{1}{R V_{0}}\left((R+1) \frac{\rho_{p}}{X_{n(2)}}-\frac{1}{X_{n}^{0}}\right)+\frac{\chi_{13}}{V_{0}^{2}}\left(\rho_{p}^{2}-\frac{\rho_{s}^{2}}{R^{2}}\right)$

$\left.-\frac{\chi_{12}+\chi_{13}-\chi_{23}}{V_{0}}\left(\left(\rho_{p}-\frac{\rho_{s}}{R}\right)-\left(\rho_{p}-\frac{\rho_{s}}{R} v_{2(1)}\right)\right)\right\}$

$\times\left((R+1)-\left(R v_{2(1)}+v_{2(2)}\right)\right)$

$-2 \chi_{12}\left(v_{2(2)}-v_{2(1)}\right)+\chi_{12}\left(v_{2(2)}^{2}-v_{2(1)}^{2}\right)=0$

Since $\rho_{p}$ (or $\left.\rho_{s}\right), \chi_{12}, \chi_{13}, \chi_{23}$, and $V_{0}$ are given in advance, $v_{2(1)} v_{2(2)}$ can be calculated from eq 22 and 23 for a given $R$ and $X_{n(2)}$. For an adequate combination of $v_{2(1)}, v_{2(2)}, R$, and $X_{n(2)}$, the partition coefficient $\sigma$ should be calculated. From $v_{2(1)}, v_{2(2)}, \sigma$, and $R$, we can calculate $g_{(2)}(X)$ (see eq 14) and accordingly, $\rho_{p}\left(\equiv \sum_{x} g_{(2)}(X)\right) . R$ can be adequately chosen so that the calculated $\rho_{p}$ agrees well within a given uncertainty with the initial $\rho_{p}$. $X_{n(2)}$ can also be determined so as to satisfy the condition: calculated $X_{n(2)}\left(\equiv X_{n(2) c}\right)=$ the assumed $X_{n(2)}\left(\equiv X_{n(2) a}\right) \cdot v_{p(1)}, v_{p(2)}$ and other parameters can be easily derived from these quantities.

\section{COMPUTER SIMULATION}

Computer simulation was carried out using the following procedure:

1. As prerequisits, the values of $g_{0}(X)(X=$ $1,2, \cdots, m ; m$, total number of components), $V_{1}^{0}, V_{3}^{0}\left(\equiv \sum_{x} g_{0}(X)=1\right), \chi_{12}, \chi_{13}, \chi_{23}$ are given in advance. The given $\rho_{p}$ is denoted as $\rho_{p}^{a}$.

2. At first, $X_{n(2)}$ is taken as $2 X_{n}^{0}$ and designated as $X_{n(2) a}$.

3. The quantities $E_{1}$ and $E_{2}$ are defined by

$$
E_{1}=\left|\frac{\rho_{p}^{c}-\rho_{p}^{a}}{\rho_{p}^{a}}\right|
$$

and

$$
E_{2}=\left|\frac{X_{n(2) c}-X_{n(2) a}}{X_{n(2) c}}\right|
$$


$\rho_{p}^{c}$ and $X_{n(2) c}$ are the calculated values.

4. Under the conditions of 1 , three values (minimum, maximum and intermediate values) of $R$ are calculated $\left(R_{\mathrm{L}}, R_{\mathrm{H}}\right.$, and $\left.R_{\mathrm{M}}\right)$. A combination of eq $10 \mathrm{~b}$ and $13 \mathrm{~b}$ yields

$$
R=\frac{V}{\rho_{p}} v_{p(2)}-1
$$

Since the relation

$$
1 \geq v_{p(2)} \geq \sum_{x} g_{0}(X) / V
$$

holds, eq 26 can be rewritten as

$$
\frac{V}{\rho_{p}}-1 \geq \frac{V_{1}^{0}+V_{3}^{0}}{\rho_{p}}=R_{\mathrm{H}} \geq R \geq \frac{\sum_{x} g_{0}(X)}{\rho_{p}}-1=R_{\mathrm{L}}
$$

and $R_{\mathrm{M}}$ is defined as

$$
R_{\mathrm{M}}=\frac{R_{\mathrm{H}}+R_{\mathrm{L}}}{2}
$$

The true $R$ should lie between $R_{\mathrm{H}}$ and $R_{\mathrm{M}}$ or between $R_{\mathrm{L}}$ and $R_{\mathrm{M}}$. Three pairs of $v_{2(1)}$ and $v_{2(2)}$ can be obtained for these $R$ values by resolving simultaneous equations (eq 22 and 23) using Newton's method. Putting the values of $v_{2(1)}$ and $v_{2(2)}$ thus obtained and $R$ into eq 21 , we can calculate three possible values of $\sigma$. For each combination of $\sigma$ and $R, v_{p(1)}, v_{p(2)}$ (by eq 13), $g_{(1)}(X), g_{(2)}(X)$ (by eq 14), and accordingly $\rho_{p}$ (this is denoted as $\rho_{p}^{c}$ ) are calculated.

5. The three values of $E_{1}$ for corresponding $R_{\mathrm{H}}, R_{\mathrm{M}}$, and $R_{\mathrm{L}}$ are compared with each other. Using the interval halving method, the true $R$ value, for which $E_{1}$ is less than 0.001 , is obtained.

6. $X_{n(2)}$ is calculated from $g_{(2)}(X) . X_{n(2) a}$ in eq 25 (at the first calculation, $X_{n(2) a}=2 X_{n}^{0}$ ) is taken as the $X_{n(2)}$ value in the preceding calculation loop. If $E_{2}$ is larger than 0.01 , we reset

$$
X_{n(2)}=\frac{X_{n(2) c}+X_{n(2) a}}{2}
$$

and the calculation steps $1-6$ are repeated until $E_{2} \leq 0.01$ is attained.

7. If a constant polymer volume fraction $\left(v_{p}^{0}\right.$, constant) is needed, we introduce the quantity $E_{3}$, as defined by

$$
E_{3}=\left|\frac{v_{p c}^{0}-v_{p}^{0}}{v_{p}^{0}}\right|
$$

where $v_{p c}^{0}$ is the calculated value of $v_{p}^{0}$.

When $E_{3}$ is larger than 0.001 , we reset $V_{1}^{0}$ value.

8. Computer $g_{(1)}(X), X_{n(1)}, X_{w(1)}, X_{w(2)}$, $v_{1(1)}, v_{1(2)}, v_{p(1)}$, and $v_{p(2)}$, respectively.

9. Steps $1-8$ correspond to one phase separation event.

This computer experiment has the following characteristics.

(1) The relative amounts of polymers remaining in the polymer-rich phase, $\rho_{p}$ are always arbitrarily changeable at constant temperature and $\chi_{i j}$ parameters are taken as constants, irrespective of $\rho_{p}$.

(2) Thus, three $\chi_{i j}$ parameters are used as initial conditions.

(3) The addition of solvent 2 to the system brings about a significant difference in $v_{p}^{0}$ and $v_{p}^{s}\left(v_{p}^{s}>v_{p}^{0}\right)$.

The main flow chart of the simulation is shown in Figure 2. The separation step is repeated as necessary.

\section{COMPUTER EXPERIMENT}

The original polymer was assumed to have the Schulz-Zimm (SZ) type molecular weight distribution $g_{0}(X)$ with the weight-average $X$, $X_{w}^{0}=300$ and the number average $X, X_{\mathrm{n}}^{0}=150$ (the suffix 0 denotes the original polymer), given by

$$
g_{0}(X)=\frac{y^{h+1}}{\Gamma(h+1)} X^{h} \exp (-y X)
$$

with

$$
y=h / X_{n}^{0}=h / 150
$$

and

$$
h=\left(\frac{X_{w}^{0}}{X_{n}^{0}}-1\right)^{-1}
$$


Phase Equilibria of Quasi-Ternary Systems I.

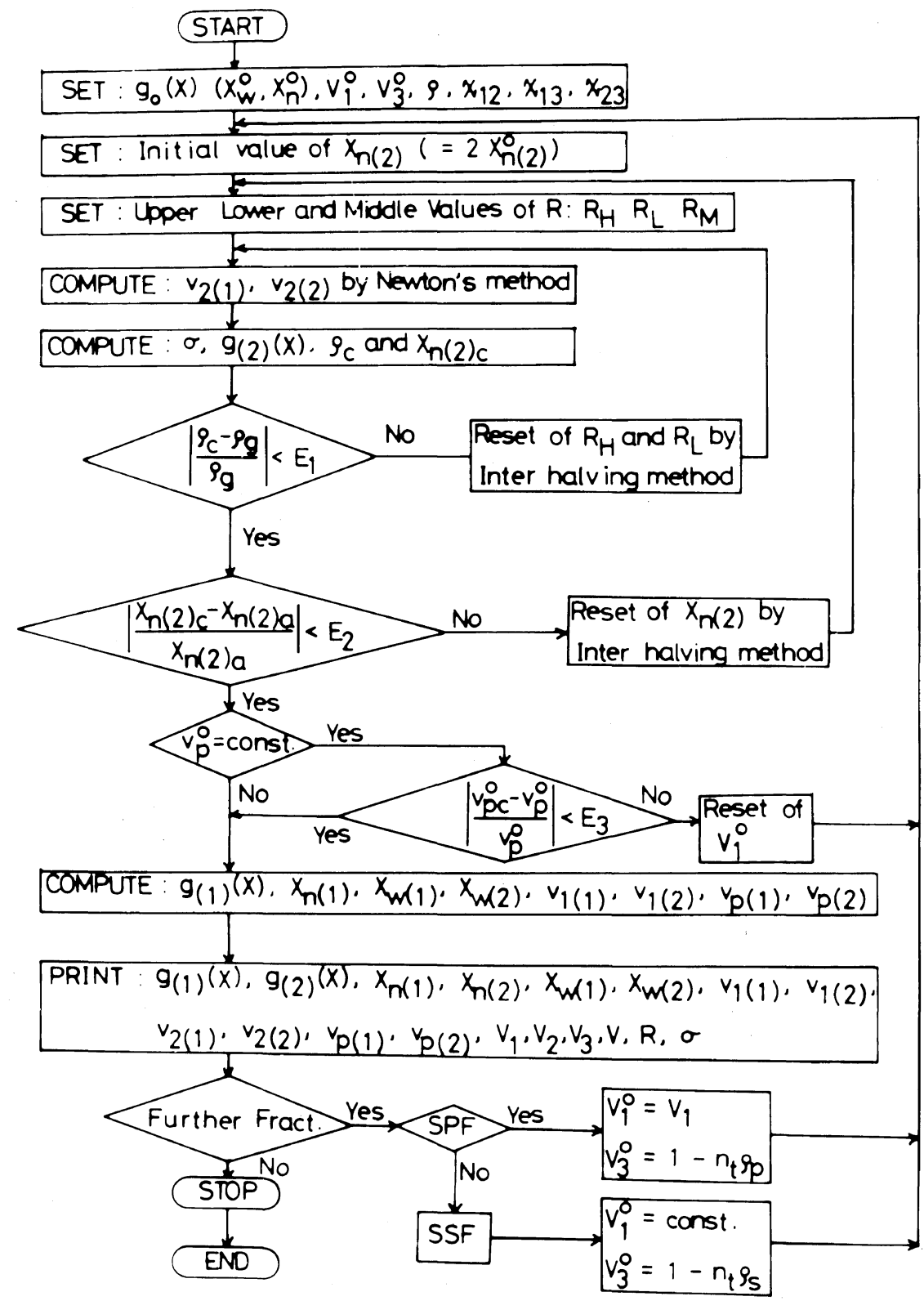

Figure 2. Flow chart of computer simulation.

$\Gamma(h+1)$, is a gamma function of $h+1, X$ is the molar volume ratio. The number of components in the original polymer was taken as 1500. $E_{1}=0.001, E_{2}=0.01$, and $E_{3}=0.001$ were employed for calculation carried out using an electric computer IBM 4341 II, under the conditions of $V_{p}^{s}=0.01$ and $\rho_{p}=1 / 15$, otherwise described.

\section{RESULTS AND DISCUSSION}

In this article, only one polymer having a specific molecular weight distribution was employed as an original polymer. The polymer 


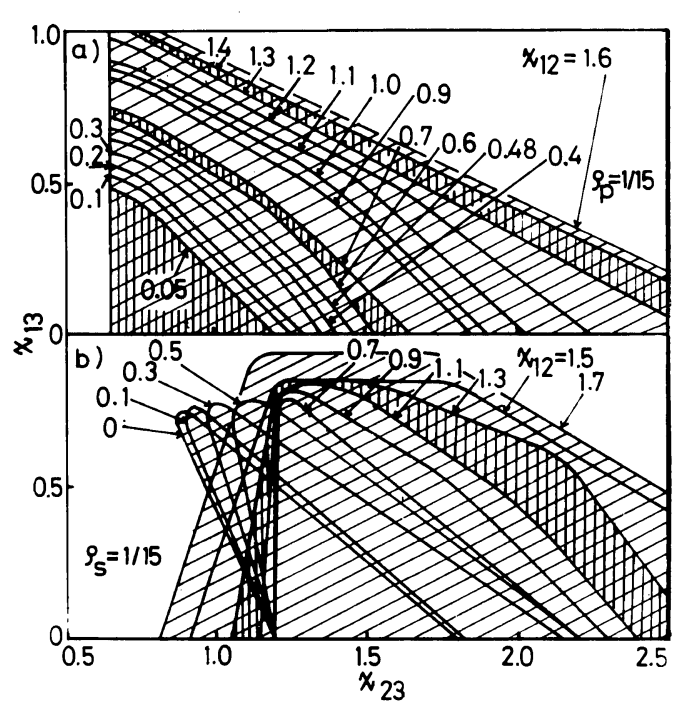

Figure 3. Allowable combination of three $\chi$ parameters, $\chi_{12}, \chi_{13}$, and $\chi_{23}$ for given conditions: the original polymer, $X_{w}^{0}=300, X_{w}^{0} / X_{n}^{0}=2.0 ; v_{p}^{s}=0.01 ; \rho_{p}=1 / 15$ in a) $\rho_{s}=1 / 15$ in b); shadowed area denotes allowed area.

with the SZ distribution $\left(X_{w}^{0} / X_{n}^{0}=2.0\right.$ and $X_{w}^{0}=300$ ) has been repeatedly used by us as the basic polymer in a series of phase separation studies for the quasi-binary system and it has been confirmed that the conclusions derived for this polymer are applicable, without extensive modification, to polymers having other molecular characteristics. The effects of the molecular characteristics of the original polymer on the phase separation features of the quasi-ternary system will be discussed in a future article. ${ }^{13}$

In an actual experiment, we often find that just any combination of solvent and nonsolvent against the polymer will not always bring about the two-phase equilibrium at a given temperature under a given pressure and that the proper choice of solvent (i.e., solvent 1) and non-solvent (i.e., solvent 2) is very important. For example, consider a case in which a polymer does not dissolve even in solvent 1 (i.e., $\chi_{13}$ is relatively large), solvents 1 and 2 are immiscible (i.e., $\chi_{12}$ is larger than 2.0) and the polymer dissolved in solvent 1 does not completely precipitate on adding an in- finite amount of solvent 2 .

In Figure 3, the shadowed area denotes the possible combination of $\chi_{12}, \chi_{13}$, and $\chi_{23}$, by which the phase separation occurs under the given conditions. When $\rho_{p}=1 / 15$ and $v_{p}^{s}=0.01$ (Figure $3 a), \chi_{23}$ should be at least larger than 0.65 . Otherwise, no phase separation will occur due to insufficient precipitation capacity of solvent 2 . The choice of solvents 1 and 2 is easier if $\chi_{12}$ is larger (i.e., solvent 1 has less thermodynamic affinity toward solvent 2 ). That is, as $\chi_{13}$ increases with a decrease in solvent power, the maximum $\chi_{23}$ value attainable becomes smaller. In this case, only a nonsolvent with weak precipitation power should be allowed to combine with solvent 1. As $\chi_{13}$ becomes smaller (i.e., the solvent power increases), phase separation will occur over a wide range of $\chi_{23}$, from weak to strong precipitation capacity. When $\rho_{s}=1 / 15$ (Figure $3 b)$, the lower limit of $\chi_{23}$, especially in the higher $\chi_{12}$ range, is much larger than 0.8 . As expected intuitively, when either the solvent power is strong (smaller $\chi_{13}$ ) or the precipitation power weak ( small $\chi_{23}$ ), the solution will not separate into two liquid phases. At $\chi_{12}=$ 0.7 , the theoretically possible lower limit of $\chi_{23}$ is 1.2, regardless of $\chi_{12}$ and this value is a maximum of the lower limits permissible for all other $\chi_{12}$ values. As is the case of $\rho_{p}=1 / 15$, large $\chi_{12}$ affords a large permissible $\chi_{13}$ and $\chi_{23}$.

Figure 4 shows the relations among the maximum $\rho_{p}$ value, $\rho_{p, \max }$ and $\chi_{23}$ for a given combination of $\chi_{12}$ and $\chi_{13}$. For smaller $\rho_{p}$, a smaller $\chi_{23}$ (less poor solvent) is permissible. At the cloud point $\left(\rho_{p}=0\right)$, minimum $\chi_{23}$ value is 0.65 . That is, an addition of a non-solvent with $\chi_{23}<0.65$ does not cause any precipitation. Large variation in $\chi_{13}$ from $0.05-0.5$ does not have any significant effect on the $\rho_{p, \max }-\chi_{23}$ curve. To precipitate the polymer completely from this solution, $\chi_{23}$ should exceed 1.5. Note that even at constant $\rho_{p}, v_{p}^{0}$ changes depending on the $\chi_{23}$ chosen.

Figure 5 illustrates the effects of $\chi_{12}, \chi_{13}$, and 
Phase Equilibria of Quasi-Ternary Systems I.

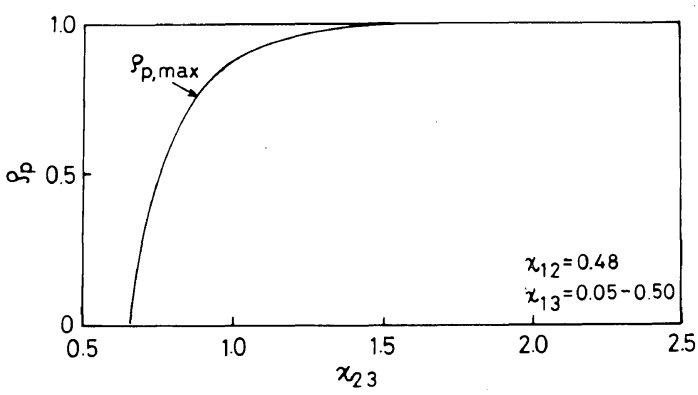

Figure 4. Relationships between the maximum $\rho_{p}$, $\rho_{p, \max }$ attainable under given conditions and $\chi_{23}$ : The original polymer, Schulz-Zimm distr., $X_{w}^{0}=300$, $X_{w}^{0} / X_{n}^{0}=2.0 ; v_{p}^{s}=0.01 ; \chi_{12}=0.48, \chi_{13}=0.05-0.50$.

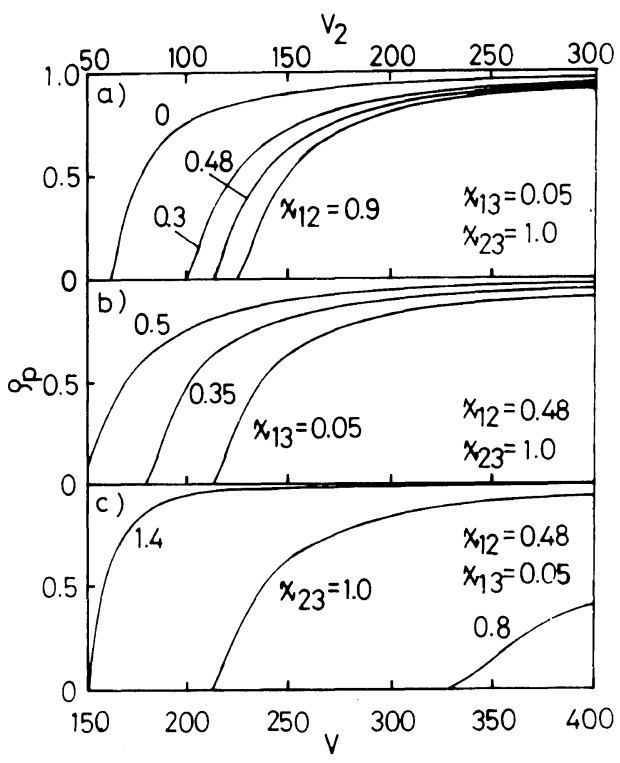

Figure 5. Plot of the relative amount of the polymer in the polymer-rich phase $\rho_{p}$ against the total volume $V$ and the volume of solvent 2: The original polymer, SchulzZimm distr., $X_{w}^{0}=300, X_{w}^{0} / X_{n}^{0}=2.0 ; v_{p}^{s}=0.01$; a) $\chi_{13}=$ $0.05, \chi_{23}=1.0$; b) $\chi_{12}=0.48, \chi_{23}=1.0$; c) $\chi_{12}=0.48, \chi_{13}=$ $0.05 ; \chi_{13}$ is shown on the curves.

$\chi_{23}$ on the relation between $\rho_{p}$ and total volume $V$. In this case, $V_{1}^{0}=100$ and $V_{3}^{0}=1$ (accordingly, $v_{p}^{s}=0.01$ ) are assumed. As $\chi_{12}$ increases, the amount of solvent 2 needed for a given $\rho_{p}$ increases. But all $V v s . \rho_{p}$ relations are superposable by shifting themselves along the horizontal axies. The effect of $\chi_{13}$ on this relation is just reverse of that of $\chi_{12}$. As $\chi_{23}$ decreases, the maximum $\rho_{p}$ value decreases and $V$ becomes large. To precipitate a given relative amount of polymer using as small an amount of solvent 2 as possible, it is necessary to use a combination of solvents 1 and 2 having small $\chi_{12}$, large $\chi_{13}$, and large $\chi_{23}$. When the mutual miscibility of solvents 1 and 2 is bad and the solvent power of solvent 1 is large and the precipitation capacity of solvent 2 is weak, we should add a large amount of solvent 2 to the quasi-binary solution of the polymer and solvent 1 , in order to bring about the phase separation. Among three $\chi$ parameters, $\chi_{23}$ plays an important role in controlling $V$. In a quasi-ternary system, $\rho_{p}$ is controlled by adding solvent 2 to the quasi-binary system (see Figure 1). As a result, the total volume increases unavoidably for larger, $\rho_{p}$, approaching the experimental limit accesible, if the same solvent 1 /solvent 2 pair is employed. For a quasi-ternary system, there are numerous combinations of solvents 1 and 2, enabling us to separate a polymer-rich phase effectively over a wide range of $\rho_{p}$. Figure 5 demonstrates that when we try to carry out a molecular weight fractionation based on the phase equilibrium phenomena of the quasi-ternary system, solvent 2 should be carefully chosen. For successive precipitation fractionation (SPF), a small $\chi_{23}$ (and, if possible, large $\chi_{13}$ ) is favorable and for successive solution fractionation (SSF), a large $\chi_{23}$ is desirable for keeping total volume as small as possible. It is a very rare case that when a drop of solvent 2 is added to the polymer/solvent 1 system, the solution becomes instantly turbid, indicating the occurrence of a phase separation. Usually, after a measurably large amount of solvent 2 is added, the cloud point of the solution (point $\mathrm{C}$ in Figure 6) is observed. Further addition of solvent 2 rapidly increases the relative amount of the polymer-rich phase $(R)$, as shown in Figure 6. In this figure, the point $\mathrm{S}$ is the composition of a starting solution whose polymer volume fraction is $v_{p}^{s}$ and by adding solvent 2 , the polymer volume fraction $v_{p}$ changes along the full line leading 


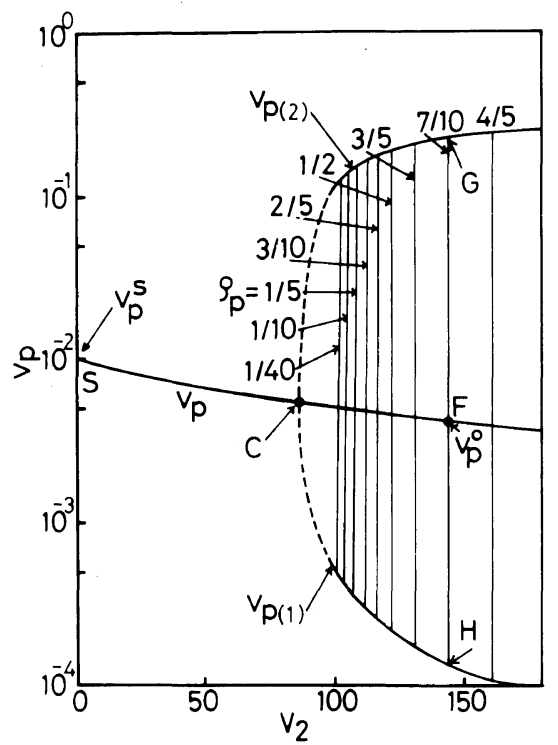

Figure 6. Change in the polymer volume fraction $v_{p}$ with the addition of solvent 2 (volume of solvent $2, V_{2}$ ) in the phase equilibrium experiment: $v_{p}^{s}$, the polymer volume fraction of the starting solution $\mathrm{S}$; F, see the text. The original polymer, Schulz-Zimm distr., $X_{w}^{0}=$ $300, X_{w}^{0} / X_{n}^{0}=2.0 ; v_{p}^{s}=0.01 ; \chi_{12}=0.48, \chi_{13}=0.20, \chi_{23}=$ 1.00 .

to the cloud point $\mathrm{C}$. Further addition of solvent 2 after the cloud point makes $\rho_{p}$ large and, for example, at point $\mathrm{F}$ a phase equilibrium is attained. A fine line passing through $\mathrm{F}$ is a tie line connecting the polymer-rich phase $G$ and the polymer-lean phase $H$. The polymer volume fraction at $\mathrm{F}$ is defined as an initial polymer volume fraction (i.e., initial "concentration") and denoted by $v_{p}^{0}$. In the small $\rho_{p}$ region, an approximation of $v_{p(1)}=0$ should not be employed when the detailed phase separation characteristics are to be evaluated.

Figures 7 and 8 show the effects of $\chi_{12}, \chi_{13}$, and $\chi_{23}$ on $v_{p(1)}$. The broken lines in the figures are the limit of the phase separation, occurring theoretically under given conditions. The full lines give the same $v_{p(1)}$ value. Figure 9 shows the effects of $\chi_{12}$ and $\chi_{23}$ on $v_{p(2)}$ at constant $\chi_{13}$. Generally speaking, $v_{p(2)}$ changes from $\chi_{23}$ dependent to $\chi_{12}$-dependent as $\chi_{23}$ increases.

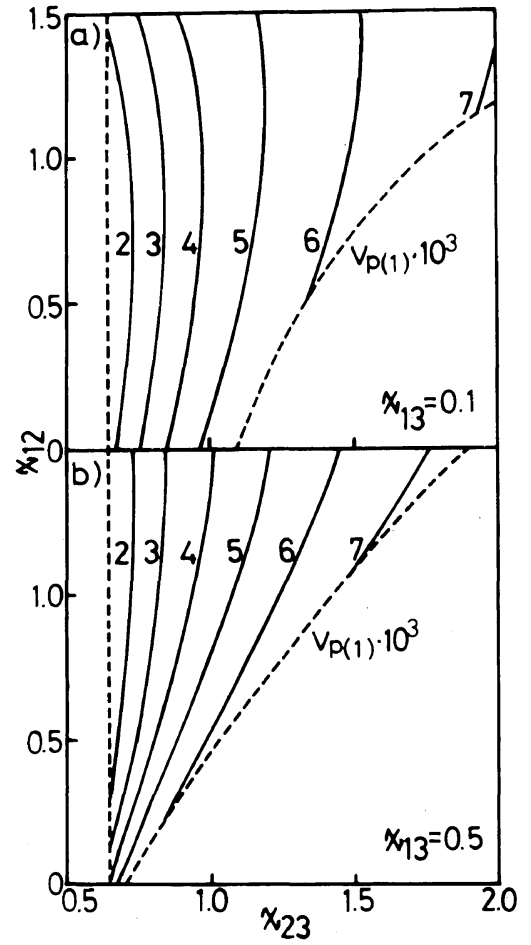

Figure 7. Relationships between $\chi_{12}, \chi_{23}$ (at a given $\chi_{13}$ ), and the polymer volume fraction in a polymerlean phase $v_{p(1)}$ : In the area between the two broken lines, a phase equilibrium is theoretically possible under given conditions. The original polymer SchulzZimm distr., $X_{w}^{0}=300, X_{w}^{0} / X_{n}^{0}=2.0 ; \rho_{p}=1 / 15 ; v_{p}^{s}=0.01$; a) $\chi_{13}=0.1$, b) $\chi_{13}=0.5$.

Occasionally, the transition point is outside the region in which the phase separation can occur.

Similar effects of $\chi_{13}$ and $\chi_{23}$ on $v_{p(2)}$ are shown in Figure 10. In the range $\chi_{23}<1.0, v_{p(2)}$ is mainly determined by $\chi_{23}$ and in the range $\chi_{23}>1.0, v_{p(2)}$ is strongly $\chi_{13}$-dependent. The broken line shows the limit within which the phase separation under given conditions is theoretically possible.

From Figures 9 and 10, it is concluded that $v_{p(2)}$ becomes larger when the mutual miscibility of solvent 1 and 2 is high and the affinity of solvent 1 to the polymer is weak and the precipitation capacity of solvent 2 is weak.

Figures 11 and 12 show the effects of $\chi_{12}$, 
Phase Equilibria of Quasi-Ternary Systems I.

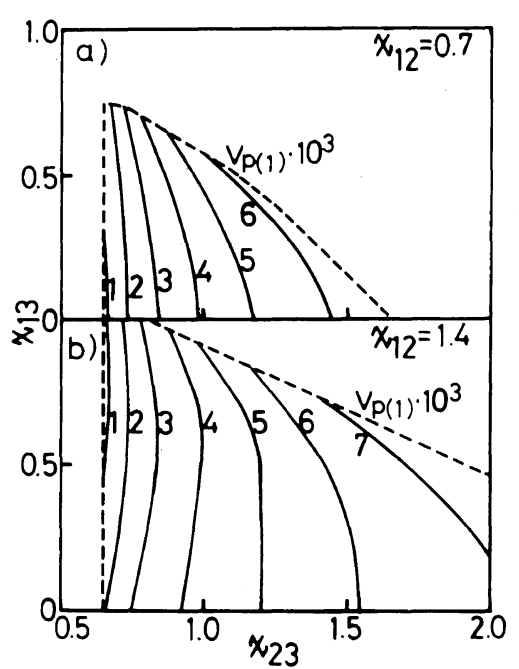

Figure 8. Relationships among $\chi_{13}, \chi_{23}$, and the polymer volume fraction in a polymer-lean phase $v_{p(1)}$ at a given $\chi_{12}(0.7$ in a) and 1.4 in $b)$ ). In the area surrounded by broken lines, a phase equilibrium under given conditions is theoretically possible. The original polymer, Schulz-Zimm distr., $X_{w}^{0}=300, X_{w}^{0} / X_{n}^{0}=2.0$; $\left.v_{p}^{s}=0.01 ; \rho_{p}=1 / 15\right)$.

$\chi_{13}$, and $\chi_{23}$ on the partition coefficient $\sigma$ and the liquid phase volume ratio $R$. Figure 11a) and c) correspond to the case of $\chi_{12}=0.7\left(\rho_{p}=\right.$ $1 / 15)$ and Figure 11b) and d) to $\chi_{12}=1.4\left(\rho_{p}=\right.$ $1 / 15)$. In the former case, both $\sigma$ and $R$ are predominantly controlled by $\chi_{23}$, regardless of $\chi_{13}$. Smaller $\chi_{23}$ yields larger $\sigma$ and larger $R$, and hence, the carefull choice of a not so poorsolvent is strongly recommended for effective fractionation. In the latter case $\left(\chi_{12}=1.4\right.$ and $\left.\rho_{p}=1 / 15\right)$, in the range $\chi_{23}<1.0$ both $\sigma$ and $R$ are $\chi_{23}$-dependent, but in the range $\chi_{23}>1.0 \sigma$ together with $R$ depend on $\chi_{23}$ as well as $\chi_{13}$. To make $\sigma$ and $R$ as large as possible, it is better to choose solvents 1 and 2 with large $\chi_{13}$ and small $\chi_{23}$ (i.e., not so good-solvent and not so poor-solvent). The use of a solvent 2 with too strong a precipitation capacity should be avoided from the standpoint of separation efficiency.

Figure 12 shows a case of $\chi_{12}=1.3$ and $\rho_{s}=$ $1 / 15$ (i.e., $\rho_{p}=14 / 15$ ). In the range $\chi_{23}<1.5$, both $\sigma$ and $R$ are mainly controlled by $\chi_{23}$

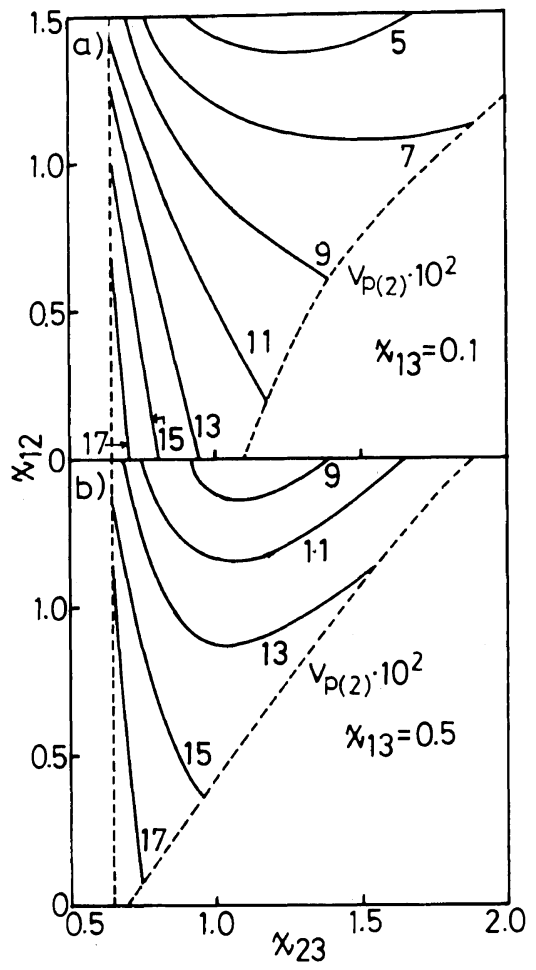

Figure 9. Relationships among $\chi_{12}, \chi_{23}$, and the polymer-volume fraction in a polymer-rich phase $v_{p(2)}$ at given $\chi_{13}(0.1$ in a), 0.5 in b)). In the area surrounded by two broken lines, a phase equilibrium is theoretically possible under given conditions; The original polymer, Schulz-Zimm distr., $X_{w}^{0}=300, X_{w}^{0} / X_{n}^{0}=2.0 ; \rho_{p}=1 / 15$; $v_{p}^{s}=0.01$.

alone and in the range $\chi_{23}>1.5, \chi_{13}$, in place of $\chi_{23}$, plays an important role in determining $\sigma$ and $R$. Regardless of $\rho_{p}$ (or $\left.\rho_{s}\right)$, the selection of solvent 2 (i.e., non-solvent) is important for keeping the fractionation efficiency as high as possible.

Figure 13 shows the effects of $\chi_{12}, \chi_{13}$, and $\chi_{23}$ on the normalized molecular weight distribution curve $g_{(2)}(X)$ of the polymer in a polymer-rich phase at $\rho_{p}=1 / 15$. A combination of solvents 1 and 2 with smaller $\chi_{12}$, larger $\chi_{13}$ and smaller $\chi_{23}$ yields a polymer with a sharp molecular weight distribution still in a polymer-rich phase. The effects of $\chi_{13}$ on $g_{(2)}(X)$ are not particularly and even negligible, but $\chi_{23}$ has a powerful effect on $g_{(2)}(X)$. 


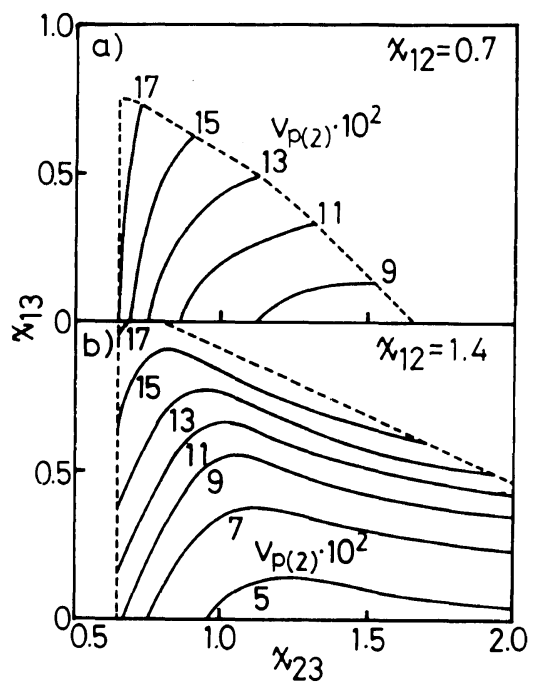

Figure 10. Relationship between $\chi_{13}, \chi_{23}$, and the polymer-volume fraction in a polymer-rich phase $v_{p(2)}$ at given $\chi_{12}(0.7$ in a) and 1.4 in $b)$ ). In the area surrounded by the broken line, a phase equilibrium can theoretically occur under given conditions. The original polymer, Schulz-Zimm distr., $X_{w}^{0}=300, X_{w}^{0} / X_{n}^{0}=2.0 ; \rho_{p}=1 / 15$; $v_{p}^{s}=0.01 ; v_{p(2)}$ is denoted on curve.

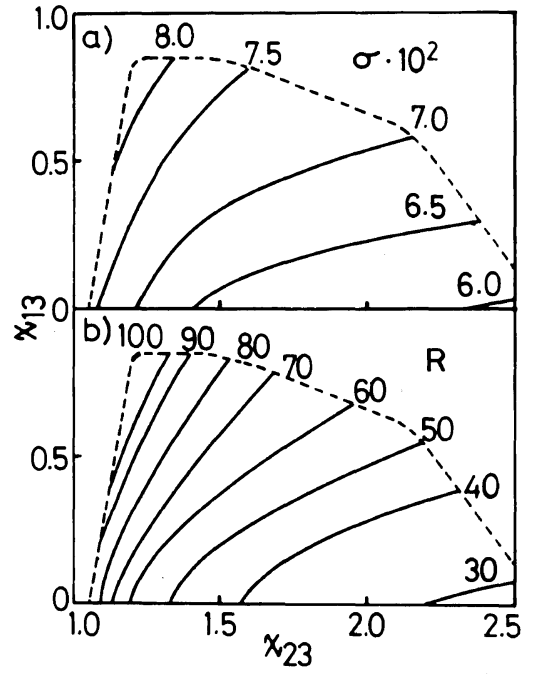

Figure 12. Correlationships between $\chi_{13}$ and $\chi_{23}$ yielding constant partition coefficient in a) or phase volume ratio $R$ in b) at $\chi_{12}=1.30$. The original polymer, SchulzZimm distr., $X_{w}^{0}=300, X_{w}^{0} / X_{n}^{0}=2.0 ; \rho_{p}=14 / 15\left(\rho_{s}=\right.$ $1 / 15) ; v_{p}^{s}=0.01$.

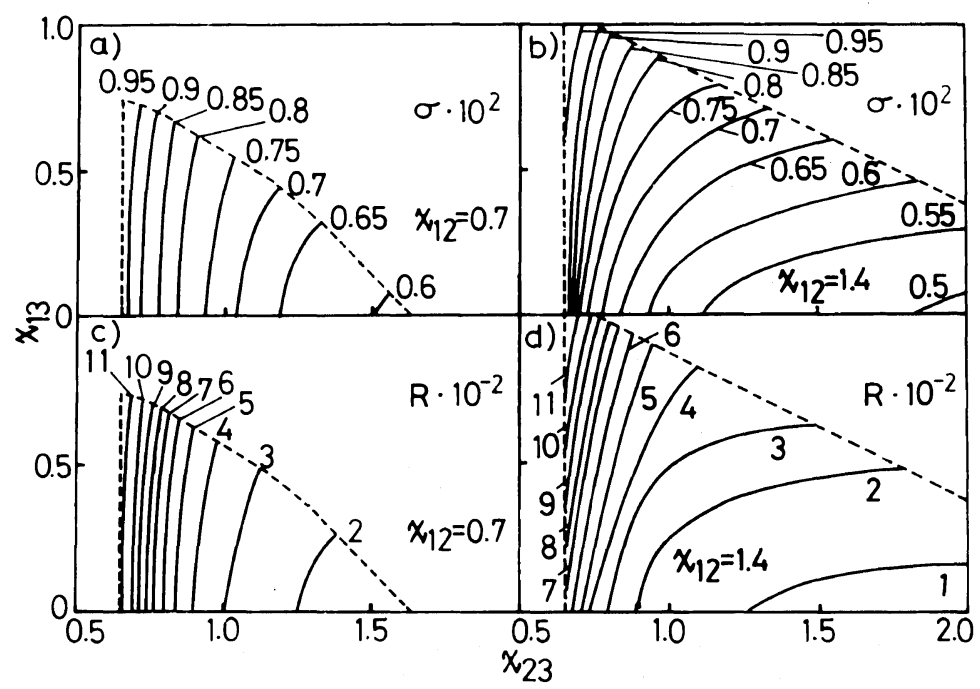

Figure 11. Correlationship between $\chi_{13}$ and $\chi_{23}$ yielding constant partition coefficient $\sigma$ or phase volume ratio $R$ at a given $\chi_{12}\left(0.7\right.$ in a) and c), 1.4 in b) and d)). The original polymer, Schulz-Zimm distr., $X_{w}^{0}=$ $300, X_{w}^{0} / X_{n}^{0}=2.0 ; \rho_{p}=1 / 15 ; v_{p}^{s}=0.01$. 


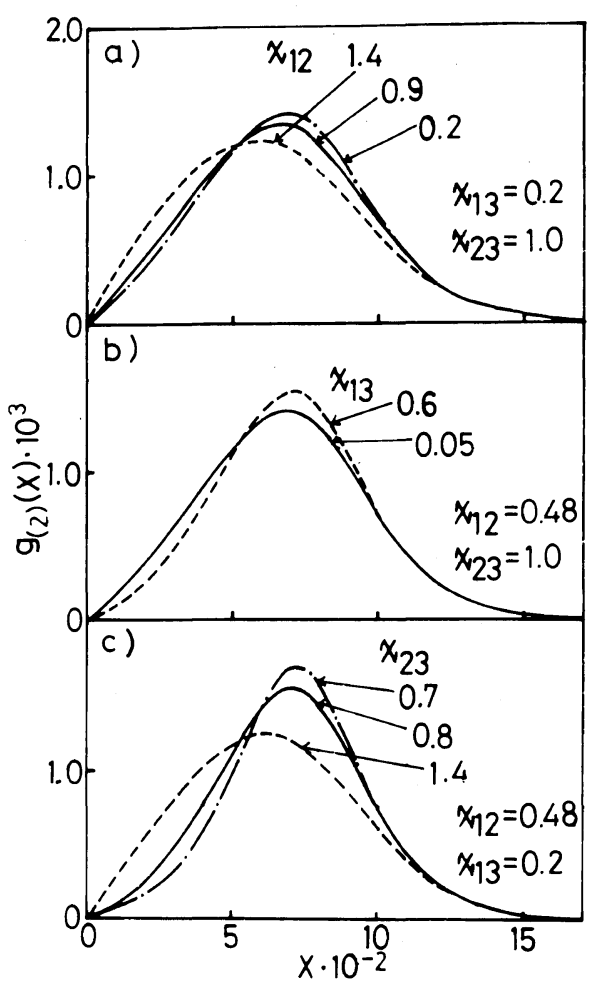

Figure 13. Effect of $\chi_{12}, \chi_{13}$, and $\chi_{23}$ parameters on the normalized molecular weight distribution curve of the polymer-rich phase $g_{(2)}(X)$, separated from a solution $\left(v_{p}^{s}=0.01\right)$ of the polymer with Schulz-Zimm distr., $\left(X_{w}^{0}=300, X_{w}^{0} / X_{n}^{0}=2.0\right): \rho_{p}=1 / 15 ;$ a) $\chi_{13}=0.2, \chi_{23}=$ 1.0 ; b) $\chi_{12}=0.48, \chi_{23}=1.0$; c) $\chi_{12}=0.48, \chi_{13}=0.2$.
Figure 14 shows the effects of $\chi_{12}, \chi_{13}$, and $\chi_{23}$ on the normalized molecular weight distribution curve $g_{(1)}(X)$ of the polymer in a polymer-lean phase at $\rho_{s}=1 / 15$ (i.e., $\rho_{p}=$ $14 / 15)$. A similar tendency to that observed in Figure 13 in the effects of three $\chi$ parameters was observed on $g_{(1)}(X)$, but these effects were not so remarkable. Therefore, in a succesive solutional fractionation run, the choice of two solvents should not be particularly importance. Kamide and coworkers noted that in a quasi-binary solution, the concentration dependence of $\chi$ parameter does not adequately influence $g_{(1)}(X) .^{14}$

Figure 15 shows the effects of $\chi_{13}$ and $\chi_{23}$ on the ratio $X_{w(2)} / X_{n(2)}$ and the standard deviation of $g_{(2)}(X), \sigma_{(2)}^{\prime}$. Both these parameters represent the breadth of the molecular weight distribution of the polymer in a polymer-rich phase. In the small $\chi_{23}$ region, the polydispersity of the polymer remaining in a polymer-rich phase is $\chi_{23}$-dependent, being smaller as $\chi_{23}$ decreases. In preparing polymer fractions by isolating the polymers from a polymer-rich phase to be separated from a quasi-ternary system, a not so poor-solvent should be used as solvent 2 .

Figure 16 shows the effects of $\chi_{13}$ and $\chi_{23}$ on

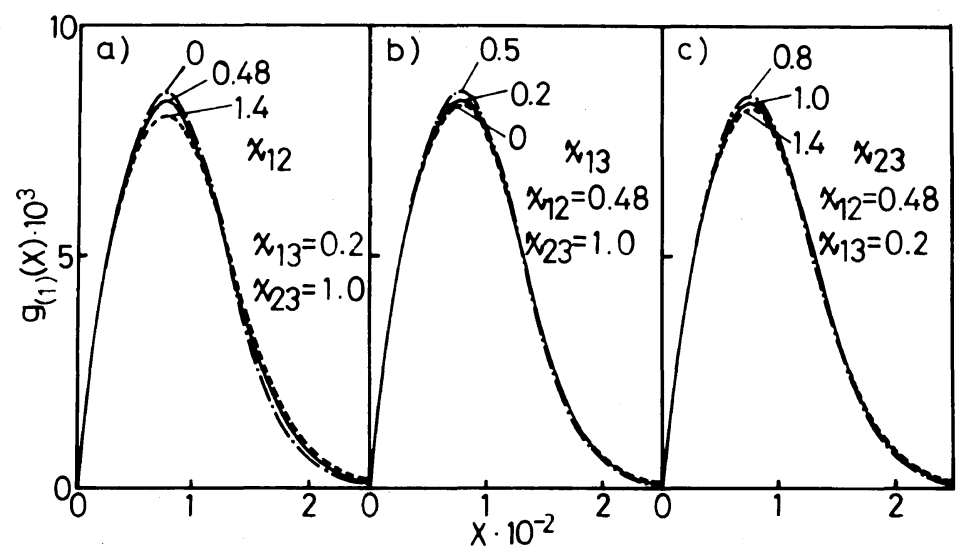

Figure 14. Effects of $\chi_{12}, \chi_{13}$, and $\chi_{23}$ parameters on the molecular weight distribution curve of the polymer-lean phase $g_{(1)}(X)$, separated from a solution $\left(v_{p}^{s}=0.01\right)$ of the polymer with Schulz-Zimm distr., $\left(X_{w}^{0}=300, X_{w}^{0} / X_{n}^{0}=2.0\right) ; \rho_{p}=14 / 15\left(\rho_{s}=1 / 15\right)$; a) $\chi_{13}=0.2, \chi_{23}=1.0 ;$ b) $\chi_{12}=0.48, \chi_{23}=1.0$; c) $\chi_{12}=0.48$, $\chi_{13}=0.2$. 


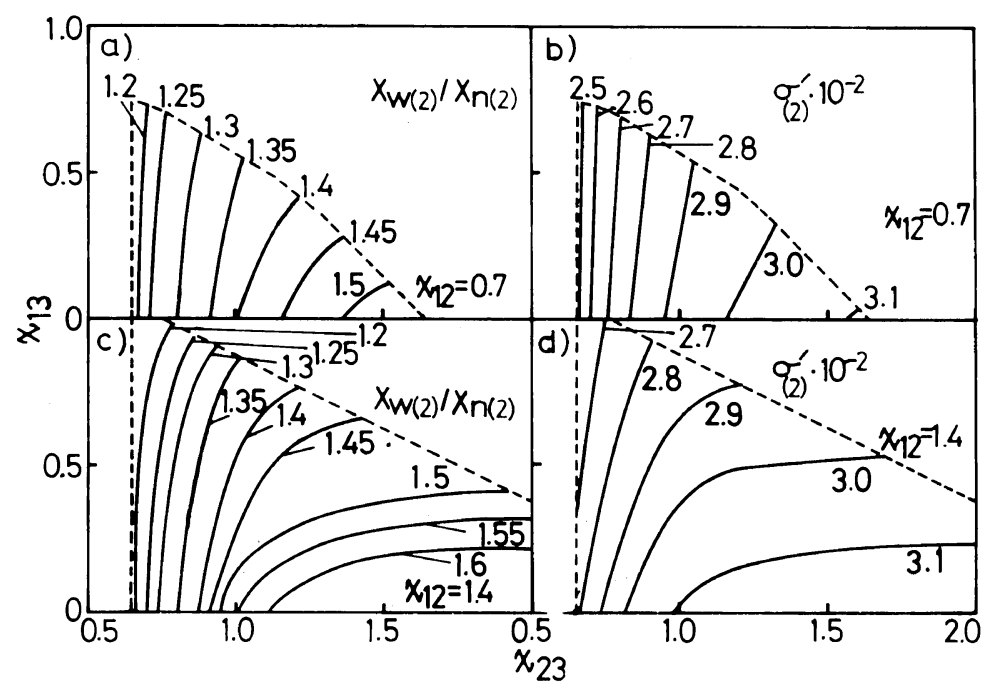

Figure 15. Correlationship between $\chi_{13}$ and $\chi_{23}$ yielding constant $X_{w(2)} / W_{n(2)}$ or the standard deviation $\sigma_{(2)}$ in the polymer-rich phase at a given $\chi_{12}(0.7$ in a) and b), 1.4 in c) and d)). The original polymer, Schulz-Zimm distr., $X_{w}^{0}=300, X_{w}^{0} / X_{n}^{0}=2.0, \sigma_{0}^{\prime}=212.2 ; \rho_{p}=1 / 15, v_{p}^{s}=0.01$.

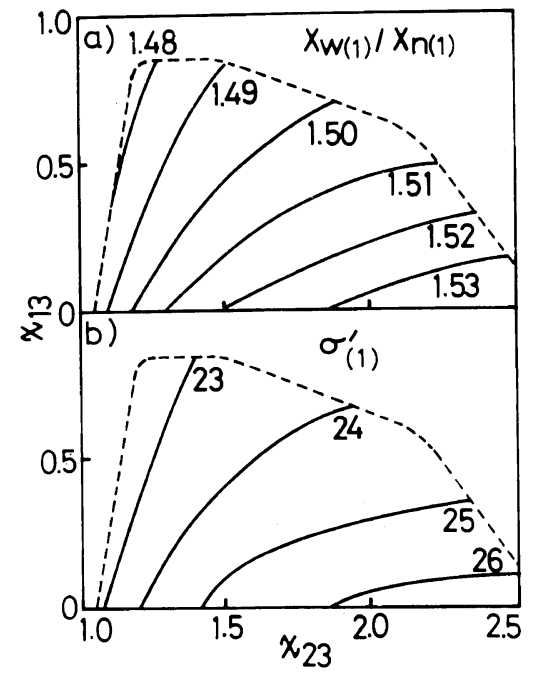

Figure 16. Correlationship between $\chi_{13}$ and $\chi_{23}$ yielding constant $X_{w(1)} / X_{n(1)}$ a) or the standard deviation $\sigma_{(1)}$ in the polymer-lean phase b) at $\chi_{12}=1.3$. The original polymer, Schulz-Zimm distr., $X_{w}^{0}=300, X_{w}^{0} / X_{n}^{0}=2.0$, $\sigma_{0}^{\prime}=212.2 ; \rho_{p}=14 / 15\left(\rho_{s}=1 / 15\right) ; v_{p}^{s}=0.01$.

the polydispersity of the polymer-lean phase. When $\chi_{12}=1.30$ and $\rho_{s}=1 / 15$ (i.e., $\rho_{p}=$ 14/15). As $\chi_{23}$ decreases, $X_{w(1)} / X_{n(1)}$ and $\sigma_{(1)}^{\prime}$ decrease very gradually, approaching limiting values (i.e., $X_{w(1)} / X_{n(1)}=1.48$ and $\sigma_{(1)}^{\prime}=22.8$ ). The effect of $\chi_{23}$ is the most predominant of the three $\chi$ parameters, but its magnitude is small, compared to the effects of the $\chi$ parameters on $X_{w(2)} / X_{n(2)}$ and $\sigma_{(2)}^{\prime}$.

Figure 17 shows the effects of the $\chi$ parameters on the phase diagram. A polymerlean phase lies almost on the solvent 1-solvent 2 axis because $v_{p(1)} \cong 0$. The coexistence curve of a polymer-rich phase side is approximately parallel to the polymer-solvent 1 axis. The tie-line connecting the two coexistence phases is, interestingly, parallel to the polymer-solvent 2 axis, particularly for larger $\chi_{12}$ and smaller $\chi_{13}$. That is, the volume fractions of solvent 1 in both phases are approximately equal, irrespective of $\rho_{p}$. An increase in $\chi_{12}$ and a decrease in $\chi_{13}$ and $\chi_{23}$ shift the coexistence curve of a polymer-rich phase to a larger $v_{2}$ region. The shape of the coexistence curves is not significantly affected by the $\chi_{12}$ and $\chi_{23}$ parameters. The role of $\chi_{13}$ at this point cannot be neglected.

Figure 18 shows the effect of the selective adsorption parameter $\theta_{2}$, defined by 
Phase Equilibria of Quasi-Ternary Systems I.
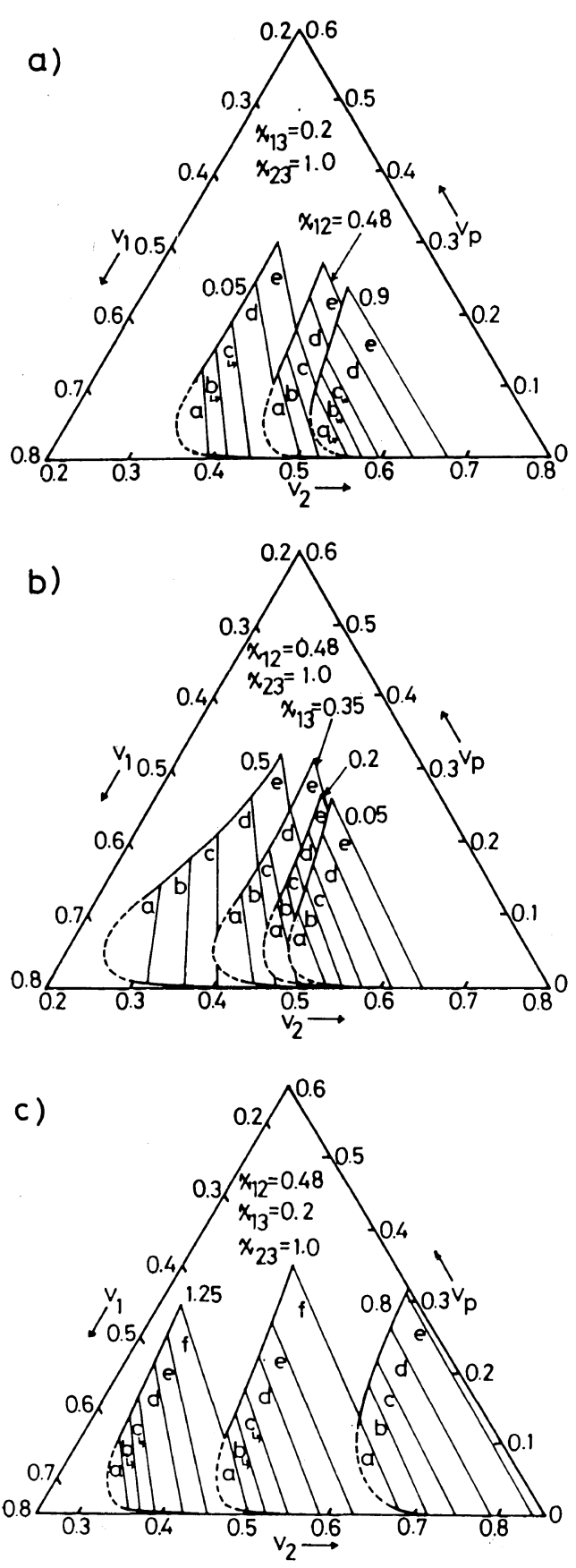

Figure 17. Effects of $\chi_{12}, \chi_{13}$, and $\chi_{23}$ parameters on the phase diagram of a quasi-ternary system of the polymer (Schulz-Zimm distr., $X_{w}^{0}=300, X_{w}^{0} / X_{n}^{0}=2.0$ ) dissolved in binary mixture: $v_{p}^{s}=0.01$; a) $\chi_{13}=0.2, \chi_{23}=$ 1.0 ; b) $\chi_{12}=0.48, \chi_{23}=1.0$; c) $\chi_{12}=0.48, \chi_{13}=0.2$; $\mathrm{a}, \rho_{p}=$ $1 / 15 ; \mathrm{b}, \rho_{p}=3 / 10 ; \mathrm{c}, \rho_{p}=1 / 2 ; \mathrm{d}, \rho_{p}=7 / 10 ; \mathrm{e}, \rho_{p}=4 / 5$; $\mathrm{f}, \rho_{p}=14 / 15$.

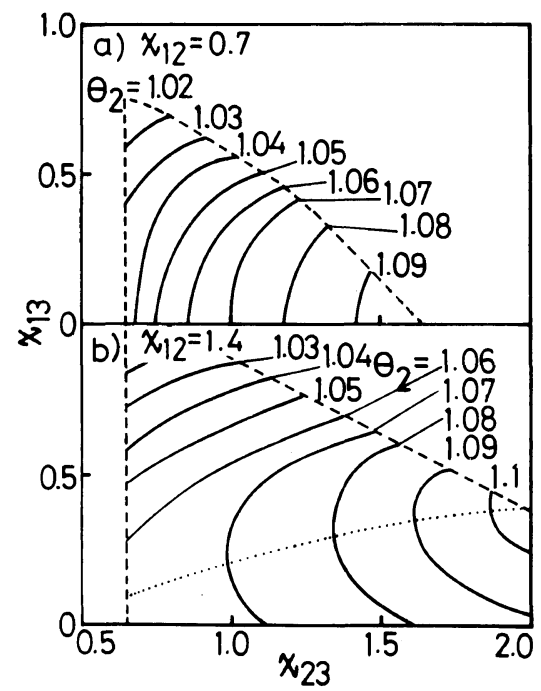

Figure 18. Correlationships between $\chi_{13}$ and $\chi_{23}$ yielding constant degree of selective adsorption $\theta_{2}$. The original polymer, Schulz-Zimm distr., $X_{w}^{0}=300$, $X_{w}^{0} / X_{n}^{0}=2.0 ; \rho_{p}=1 / 15 ; v_{p}^{0}=0.01 ;$ a) $\chi_{12}=0.7$, b) $\chi_{12}=$ 1.4 .

$$
\theta_{2}=\frac{v_{2(1)} /\left(v_{1(1)}+v_{2(1)}\right)}{v_{2(2)} /\left(v_{1(2)}+v_{2(2)}\right)}
$$

on combining three $\chi_{i j}$ parameters. The number in the figure denotes the $\theta_{2}$ value and the full lines are contour lines giving the same $\theta_{2}$ value. When $\chi_{12}=0.7$, a constant $\theta_{2}$ line is mainly controlled by $\chi_{23}$ and when $\chi_{12}=1.4$, the constant $\theta_{2}$ line changes from $\chi_{13^{-}}$ dependent to $\chi_{23}$-dependent. In general, the selective adsorption becomes large for a large $\chi_{23}$ region, that is, when a very poor solvent is used. It should be noted that for large $\chi_{23}$, only low $\chi_{13}$ (i.e., very good solvent) is permitted. Thus, a large selective adsorption should theoretically occur for a polymer-very good solvent-very poor solvent combination.

The effects of the three parameters $\chi_{12}, \chi_{13}$, and $\chi_{23}$ on the various characteristic parameters of the phase equilibrium are summarized as follows: A decrease in $\chi_{12}$ and $\chi_{23}$ and an increase in $\chi_{13}$, are accompanied by an increase in $\sigma, R, v_{p(1)}$, and $v_{p(2)}$ and a decrease in $X_{w(1)} / X_{n(1)}$ and $X_{w(2)} / X_{n(2)}$. 


\section{K. Kamide, S. Matsuda, and Y. Miyazaki}

\section{REFERENCES}

1. See, for example, K. Kamide, "Fractionation of Synthetic Polymers," L. H. Tung, Ed., Marcel Dekker Inc., New York, 1977, Chapter 2; K. Kamide and Y. Miyazaki, Polym. J., 9, 61 (1977); K. Kamide, Y. Miyazaki, and T. Abe, Polym. J., 9, 395 (1977); I. Noda, H. Ishikawa, Y. Miyazaki, and K. Kamide, Polym. J., 12, 87 (1980); K. Kamide, K. Sugamiya, T. Kawai, and Y. Miyazaki, Polym. J., 12, 67 (1980); K. Kamide and Y. Miyazaki, Polym. J., 12, 153 (1980); K. Kamide and Y. Miyazaki, Polym. J., 12, 205 (1980); K. Kamide and Y. Miyazaki, Polym. J., 13, 325 (1981); K. Kamide, T. Abe, and Y. Miyazaki, Polym. J., 14, 355 (1982).

2. See, for example, R. Koningsveld and A. J. Staverman, J. Polym. Sci., A-2, 6, 305 (1968); R. Koningsveld and A. J. Staverman, J. Polym. Sci., A-
2, 6, 325 (1968); R. Koningsveld, Adv. Polym. Sci., 7, $1(1970)$.

3. P. J. Flory, J. Chem. Phys., 12, 425 (1944).

4. R. S. Scott, J. Chem. Phys., 13, 178 (1945).

5. R. S. Scott, Trans. Faraday Soc., 45, 1142 (1949).

6. M. Nakagaki and H. Sunada, Yakugaku Zasshi, 83, 1147 (1963).

7. H. Tompa, Trnas. Faraday Soc., 45, 1142 (1949).

8. W. R. Krigbaum and D. K. Carpenter, J. Polym. Sci., 14, 241 (1954).

9. K. W. Suh and D. W. Liou, J. Polym. Sci., $A-2,6$, 813 (1968).

10. A. Münster, J. Polym. Sci., 5, 333 (1949).

11. H. Okamoto, J. Polym. Sci., 33, 507 (1958).

12. R. Koningsveld, Discuss. Faraday Soc., 49, 144 (1970).

13. K. Kamide and S. Matsuda, Polym. J., in press.

14. K. Kamide and K. Sugamiya, Makromol. Chem., 156, 259 (1972). 